\title{
Horizons in Novel Oral Anticoagulation Therapy in Concomitant Acute Coronary Syndromes and Atrial Fibrillation
}

Leydimar Anmad Shihadeh, Diego Fernández-Rodríguez*, Javier Lorenzo-González and Julio Hernández-Afonso

Cardiology Department, Nuestra Señora de Candelaria University Hospital, Santa Cruz de Tenerife, Spain

\begin{abstract}
Thrombus formation and coronary artery occlusion, in acute coronary syndromes, occur as a result of an atherosclerotic plaque rupture/erosion and the subsequent activation of platelets and coagulation factors. Also, cardioembolic events, in atrial fibrillation, are related to the thrombus formation and the systemic arterial embolization secondary to the blood stasis in left atrium.

Antiplatelet treatments in acute coronary syndromes and long-term oral anticoagulation in atrial fibrillation have improved prognosis by reducing ischemic events but both treatments are associated with an increase in the risk of bleeding. Furthermore, thrombin and activated factor $X$ are the key elements in the coagulation cascade and novel oral anticoagulants act by inhibiting these coagulation factors, generating a double effect: the reduction of ischemic events and the increment in hemorrhagic events.

To date, the clinical benefit of novel oral anticoagulants, in patients presenting acute coronary syndromes and atrial fibrillation, has not well studied. For that reason, the objective of this manuscript is to explain basic clinical trials testing novel oral anticoagulants in patients with acute coronary syndromes and ongoing trials evaluating the use of new oral anticoagulants in population with acute coronary syndromes and atrial fibrillation: the PIONEER AF-PCI (Rivaroxaban), the RT-AF (Rivaroxaban) and the REDUAL-PCI (Dabigatran) trials.
\end{abstract}

Keywords: Acute coronary syndromes; Atrial fibrillation; Thrombin; Factor Xa; New oral anticoagulants

\section{Novel Oral Anticoagulants After Acute Coronary Syndromes}

The ischemic risk in patients with acute coronary syndromes (ACS) persists after hospital discharge, even with proper antithrombotic therapy. The rate of cardiovascular death, non-fatal myocardial infarction (MI) or stroke during the first year after hospitalization is around $10 \%$, despite antithrombotic and revascularization therapies [1-3].

Multiple clinical trials have been conducted to assess the benefit of classic anticoagulants after ACS. The WARIS-II study [4] showed a reduction in reinfarction and all-cause mortality in patients surviving a MI who were treated with warfarin. Despite the reduction in cardiovascular events, the treatment with classic anticoagulants at longterm was not implemented in clinical practice due to the increase in bleeding, the multiple drug interactions and the need for strict control.

Recently, the WOEST study [5] randomized 573 patients receiving classic anticoagulants who underwent to percutaneous coronary intervention (PCI) to clopidogrel alone (double therapy) or clopidogrel plus aspirin (triple therapy). No increase in thrombotic events was observed in double therapy group in comparison with triple therapy. There were a reduction in the primary endpoint (any bleeding episode within one year after PCI) in double therapy group compared with triple therapy group ( $19.4 \%$ for double therapy vs. $44.4 \%$ for triple therapy (hazard ratio (HR) [95\% confidence interval (CI)]: 0.36 [0.260.50], $\mathrm{p}<0.0001)$.

In spite of the reduction under double therapy, long-term treatment with conventional anticoagulants was associated with a high rate of bleeding. It is also noteworthy that after ACS, thrombin and activated Factor X (FXa) remain high at long-term4. For these reasons, novel oral anticoagulants (NOAC) are being studied as alternative treatments for patients who have overcome the acute phase of an ACS.
NOAC selectively inhibits thrombin or FXa (Figure 1 and Table 1) $[6,7]$. Direct thrombin inhibitors (DTI) are attractive because they directly bind the active site of the thrombin, generating the subsequent inhibition of thrombin and soluble fibrin. FXa is another attractive target because it is at the beginning of the common coagulation pathway. Some groups have postulated that the selective inhibition of coagulation factors above thrombin could present an optimal riskbenefit balance, because a few quantity of coagulation factors escape from inhibition allowing that small amounts of thrombin can still be generated, and therefore the treatment with FXa inhibitors may protect against ischemic events without greatly increase in the risk of bleeding [6,7].

\section{Direct thrombin inhibitors}

Ximelagatran was the first drug of a new generation of oral anticoagulants being studied in ACS setting. The ESTEEM [8] was a phase II, randomized, placebo-controlled trial that evaluated 1883 patients with ST-segment elevation MI or non-ST-segment elevation MI. The treatment was initiated within 14 days after the event and prolonged for six months. The patients were randomized to receive placebo or Ximelagatran at different doses twice daily (bid) (24 mg, 36

*Corresponding author: Diego Fernández-Rodríguez, MD, Cardiology Department, Nuestra Señora de Candelaria University Hospital, Santa Cruz de Tenerife, Spain, Tel: +34 922602 000; Fax +34 922602 009; E-mail: d.fernan.2@hotmail.com

Received August 07, 2015; Accepted August 31, 2015; Published September 06, 2015

Citation: Shihadeh LA, Fernández-Rodríguez D, Lorenzo-González J, HernándezAfonso J (2015) Horizons in Novel Oral Anticoagulation Therapy in Concomitant Acute Coronary Syndromes and Atrial Fibrillation. Cardiol Pharmacol 4: 155 doi:10.4172/2329-6607.1000155

Copyright: (c) 2015 Shihadeh LA et al. This is an open-access article distributed under the terms of the Creative Commons Attribution License, which permits unrestricted use, distribution, and reproduction in any medium, provided the original author and source are credited. 


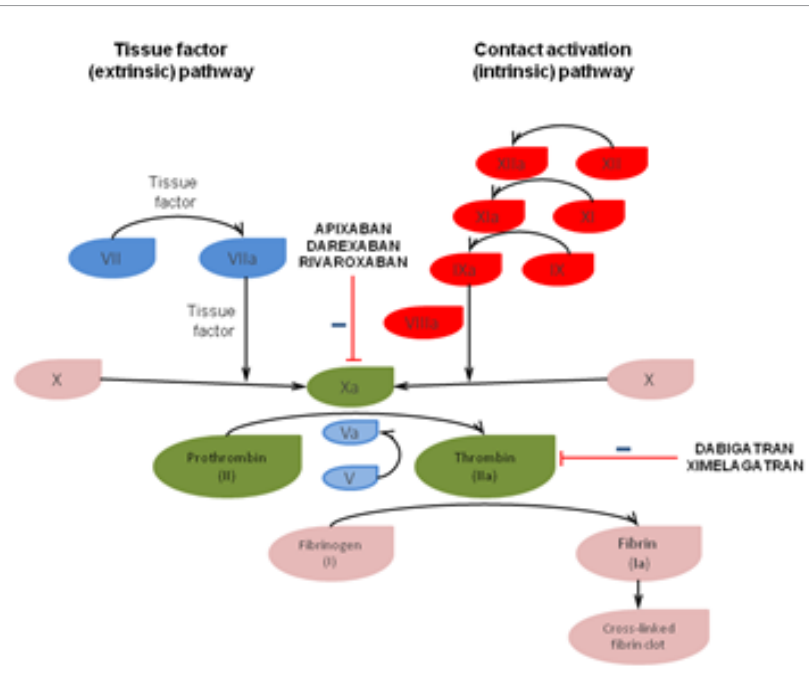

Figure 1: Therapeutic targets of NOAC. NOAC: Novel Oral AntiCoagulants.

$\mathrm{mg}$, $48 \mathrm{mg}$ or $60 \mathrm{mg}$ ) in combination with low-dose of aspirin.

The primary endpoint was a composite including all-cause death, non-fatal MI or severe recurrent ischemia showing a significant reduction in the primary endpoint when all treatment groups were grouped and compared to placebo (12.7\% for combined Ximelagatran groups vs. $16.3 \%$ for placebo; HR [95\% CI]: 0.76 [0.59-0.98]). There were no significant differences between treatment doses. Regarding secondary safety endpoints, the rate of major hemorrhagic events was very low, with no significant differences between groups $(1.8 \%$ for combined Ximelagatran groups vs. $0.9 \%$ for placebo; HR [ $95 \% \mathrm{CI}]$ : $1.97[0.80-4.84])$.

Later, Ximelagatran did not receive the Food and Drug Administration approval due to cases of severe hepatic toxicity, and therefore it was not marketed [9].

Dabigatran Etexilate was tested in the RE-DEEM trial [10], a phase II, randomized, placebo-controlled, dose-ranging study evaluating the safety of Dabigatran. The RE-DEEM included 1861 patients with STsegment elevation MI or non-ST-segment elevation MI. The treatment was initiated on average 7.5 days after the event and continued for six months.

The patients were randomized to receive placebo or different doses of Dabigatran bid (50 mg, $75 \mathrm{mg}, 110 \mathrm{mg}, 150 \mathrm{mg}$ ). Almost all patients (99.2\%) were under dual antiplatelet therapy with low-dose aspirin and clopidogrel. The primary endpoint was a composite of major bleeding or clinically relevant minor bleeding. A dose-dependent increase in primary endpoint with Dabigatran in comparison with placebo was observed (HR [95\% CI]: 1.77 [0.70-4.50] for $50 \mathrm{mg} ; 2.17$ [0.88-5.31] for $75 \mathrm{mg}$; 3.92 [1.72-8.95] for $110 \mathrm{mg}$; and 4.27 [1.86-9.81] for $150 \mathrm{mg}$ ). Respect to secondary efficacy endpoints, the combined rate of death, MI or stroke was 3.8\% in placebo group compared with $4.6 \%$ in $50 \mathrm{mg}$, $4.9 \%$ in $75 \mathrm{mg}, 3.0 \%$ in $110 \mathrm{mg}$ and $3.5 \%$ in $150 \mathrm{mg}$ Dabigatran group.

Furthermore, a substudy of the RE-LY trial [11] evaluated the efficacy and safety of Dabigatran compared to Warfarin in patients with non-valvular atrial fibrillation (AF) receiving concomitant antiplatelet therapies (not specifically ACS patients). From a total of 18113 patients included in the RE-LY trial, 6952 patients (38.4\%) received concomitant aspirin or clopidogrel at some time during the study. The absolute risks of thrombotic and hemorrhagic events were lower with Dabigatran 110 mg bid in comparison with Dabigatran 150 mg bid or Warfarin.

In comparison with Warfarin, Dabigatran $110 \mathrm{mg}$ presented similar risks for stroke and systemic embolism (HR [95\% CI]: 0.93 [0.70-1.25] for patients with antiplatelet treatment vs. 0.87 [0.66-1.15] for patients without antiplatelet treatment; interaction $\mathrm{p}=0.738)$; and lower risk for major bleeding (HR [95\% CI]: 0.82 [0.67-1.00] for patients who used antiplatelets vs. 0.79 [0.64-0.96] for patients who did not; interaction $\mathrm{p}=0.794$ ), whether patients received antiplatelet therapy or not.

\section{Factor Xa inhibitors}

Apixaban was evaluated in the APPRAISE-2 trial [12], a phase III, randomized, placebo-controlled study evaluating patients with highrisk ACS. Almost all patients were under aspirin treatment (97\%) and the majority was receiving dual antiplatelet therapy with low-dose aspirin and P2Y12 inhibitors (81\%), predominantly clopidogrel.

The treatment was initiated within the first seven days after the ACS and subjects were randomized to Apixaban 5-mg bid or placebo. The study was terminated prematurely after recruiting 7392 patients due to an increase in major bleeding related with Apixaban. The mean follow-up period was 241 days. There were no differences in the primary efficacy endpoint (combination of cardiovascular death, MI or ischemic stroke) (7.5\% for Apixaban vs. $7.9 \%$ for placebo; HR [95\% CI]: 0.95 [0.80-1.11]). The primary safety endpoint was major bleeding (1.3\% for Apixaban vs. 0.5\% for placebo; HR [95\% CI]: 2.59 [1.50-4.46]). Furthermore, a greater number of intracranial and fatal bleeding events occurred with Apixaban as compared with placebo.

Darexaban has been tested in the RUBY-1 trial [13]. The RUBY1 trial was a phase II, randomized, placebo-controlled, dose-ranging study that recruited 1279 patients presenting ST-segment elevation MI or high-risk non-ST-segment elevation MI. The treatment was initiated the first week after the event and prolonged for 26 weeks.

Patients were randomized to receive placebo or Darexaban at different doses ( $5 \mathrm{mg}$ bid, $10 \mathrm{mg}$ once daily [od], $15 \mathrm{mg}$ bid, $30 \mathrm{mg}$ od, $30 \mathrm{md}$ bid, or $60 \mathrm{mg}$ od) in combination with dual antiplatelet therapy. The primary endpoint was a composite of major bleeding or clinically relevant minor bleeding. The combined analysis of Darexaban showed an increase in hemorrhagic risk (HR [95\% CI]: 2.275 [1.13-4.60]) as compared to the bleeding rate in placebo group (3.1\%). A doseresponse relationship ( $p=0.009)$ was observed for increased bleeding with incremental Darexaban doses. No significant decrease was demonstrated in secondary efficacy endpoint (combination of death, stroke, MI, systemic embolism and severe recurrent ischemia) with Darexaban and an even an increment in this endpoint was observed with $30 \mathrm{mg}$ and $60 \mathrm{mg}$ doses.

Rivaroxaban was investigated in ATLAS ACS 2-TIMI 51 studies [14]. 15526 patients were randomized to receive Rivaroxaban at doses of (2.5-mg or 5-mg) bid or placebo (scheme 1:1:1) for a mean of 13 months and up to 31 months. This trial was a phase III, randomized, placebo-controlled, double-blind trial evaluating Rivaroxaban in patients with recent ACS. All patients received standard medical therapy including low-dose aspirin $(98.7 \%$ in $2.5 \mathrm{mg}$ group and $98.5 \%$ in $5 \mathrm{mg}$ group) and thienopyridines: clopidogrel or ticlopidine $(92.6 \%$ in $2.5 \mathrm{mg}$ group and $93 \%$ in the $5 \mathrm{mg}$ ). The primary efficacy endpoint was a combination of cardiovascular death, MI or stroke. A significant reduction in the combined analysis of Rivaroxaban doses in comparison with placebo was observed ( $8.9 \%$ for combined Rivaroxaban groups vs. 


\begin{tabular}{|c|c|c|c|c|c|}
\hline Anticoagulants & Target & Pro-drug & Bioavailability & Half-life & Renal excretion \\
\hline Ximelagatran & Thrombin & Yes & $23 \%$ & $4-5$ hours & $80 \%$ \\
\hline Dabigatran & Thrombin & Yes & $6.5 \%$ & $9-13$ hours & No \\
\hline Apixaban & FXa & No & $66 \%$ & $8-15$ hours & No \\
\hline Darexaban & FXa & No & $50 \%$ & $9-11$ hours & No \\
\hline Rivaroxaban & FXa & No & $80 \%$ & $7-11$ hours & No \\
\hline
\end{tabular}

Table 1: Pharmacokinetics of long-term NOAC tested in ACS and AF. Ximelagatran was not approved by FDA for medical use due to cases of severe hepatic toxicity; NOAC: Novel Oral Anti-Coagulants; ACS: Acute Coronary Syndromes; AF: Atrial Fibrillation; FXa: Activated Factor X; FDA: Food and Drug Administration.

$10.7 \%$ for placebo; HR [95\% CI]: 0.84 [0.74-0.96]), as well as individual Rivaroxaban doses (9.1\% for 2.5 -mg Rivaroxaban vs. $10.7 \%$ for placebo; $\mathrm{p}=0.02)$ and ( $8.8 \%$ for $5 \mathrm{mg}$ Rivaroxaban vs. $10.7 \%$ for placebo, $\mathrm{p}=0.03$ ). Furthermore, $2.5 \mathrm{mg}$ Rivaroxaban reduced death from cardiovascular causes $(2.7 \%$ for $2.5 \mathrm{mg}$ Rivaroxaban vs. $4.1 \%$ for placebo; $\mathrm{p}=0.002)$ and death from any cause $(2.9 \%$ for $2.5 \mathrm{mg}$ Rivaroxaban vs. $4.5 \%$ for placebo; $p=0.002$ ). This benefit in survival was not observed with $5 \mathrm{mg}$ Rivaroxaban. With regard to safety, the combined analysis of Rivaroxaban showed an increase in major bleeding $(2.1 \%$ for combined Rivaroxaban group vs. $0.6 \%$ for placebo; $\mathrm{p}<0.001)$ and intracranial hemorrhage $(0.6 \%$ for combined Rivaroxaban groups vs. $0.2 \%$ for placebo; $\mathrm{p}=0.009)$ but no significant increase in fatal bleeding $(0.3 \%$ for combined Rivaroxaban group vs. $0.2 \%$ for placebo; $p=0.66$ ) in comparison with placebo.

It was also noted in Rivaroxaban groups that $2.5 \mathrm{mg}$ dose showed a lower rate of fatal bleeding as compared to $5 \mathrm{mg}$ dose the dose of $(0.1 \%$ for $2.5 \mathrm{mg}$ Rivaroxaban vs. $0.4 \%$ for $5 \mathrm{mg}$ Rivaroxaban; $\mathrm{p}=0.04$ ).

\section{Ongoing Trials in Concomitant Acute Coronary Syndromes and Atrial Fibrillation}

Rivaroxaban is being tested in the PIONEER AF-PCI [15] (NCT01830543) and RT-AF [16] (NCT02334254) studies that compare Ribaroxaban vs. vitamin K Antagonists (VKA) in patients with nonvalvular AF undergoing percutaneous coronary intervention with stent implantation (bare metal or drug-eluting stents).

The PIONEER AF-PCI is a randomized, multicentric, clinical trial evaluating the safety of three different treatment strategies recruiting approximately 2,100 patients (including ACS or stable angina) in a 1:1:1 scheme to receive:

a) Rivaroxaban $15 \mathrm{mg}$ once-daily treatment strategy: Rivaroxaban $15 \mathrm{mg}$ od plus P2Y12 inhibitor (clopidogrel $75 \mathrm{mg}$, ticagrelor $90 \mathrm{mg}$ or prasugrel $10 \mathrm{mg}$ ) for 12 months.

b) Rivaroxaban $2.5 \mathrm{mg}$ twice-daily treatment strategy: Rivaroxaban $2.5 \mathrm{mg}$ bid plus dual antiplatelet therapy at investigator discretion $(1,6$ or 12 months) with low-dose aspirin and P2Y12 inhibitor (clopidogrel $75 \mathrm{mg}$, ticagrelor $90 \mathrm{mg}$ or prasugrel $10 \mathrm{mg}$ ) followed by Rivaroxaban $15 \mathrm{mg}$ od plus low-dose aspirin until completing 12 months

c) Dose-adjusted VKA treatment strategy: VKA (International Normalized Ratio [INR] 2.0-3.0) plus double antiplatelet therapy at investigator discretion (1, 6 or 12 months) with low-dose aspirin and P2Y12 inhibitor (clopidogrel $75 \mathrm{mg}$, ticagrelor $90 \mathrm{mg}$ or prasugrel 10 $\mathrm{mg}$ ) followed by VKA plus aspirin at low doses until the twelfth month.

The primary endpoint (clinically significant bleeding) is a composite of the rate of TIMI major bleeding [17], bleeding requiring medical attention, and minor bleeding at 12-months. Secondary endpoints include the incidence of each component of the composite clinically significant bleeding (TIMI major bleeding, bleeding requiring medical attention and minor bleeding), the composite of adverse cardiovascular events (cardiovascular death, MI, and stroke), as well as cardiovascular death, MI, stroke, and stent thrombosis.

The RT-AF study is a randomized, clinical trial enrolling patients in five centres comparing the safety of two therapeutic strategies recruiting approximately 420 patients (with ACS or stable angina). The endpoints will be evaluated at 12-month follow-up. The two treatment arms are:

a) Rivaroxaban plus Ticagrelor strategy: Rivaroxaban 2.5 or $5 \mathrm{mg}$ od (depending on patient clinical characteristics) plus ticagrelor $90 \mathrm{mg}$ for 12 months. Ticagrelor therapy is mandatory at least 1 month after bare metal stents and 6 months after drug-eluting stents.

c) Triple antithrombotic therapy strategy: Dose-adjusted VKA (INR 1.8-2.5) plus dual antiplatelet treatment with low doses of aspirin and clopidogrel $75 \mathrm{mg}$.

The primary safety end point is the rate of the composite of ISTH major bleeding [18] or clinically relevant non-major bleeding. Secondary endpoints include efficacy items like the rate of the composite of all-cause death, MI, stent thrombosis or ischemic stroke, as well as the same individual endpoints.

Dabigatran Etexilate is being tested in REDUAL-PCI study [19] (NCT02164864), assessing the efficacy and the safety of three strategies of antithrombotic therapy in patients with $\mathrm{AF}$ and ischemic heart disease (ACS or stable angina) that undergo to stent placement with bare metal or drug-eluting stents.

The REDUAL-PCI study is a randomized, multicentric, clinical trial that includes approximately 8,250 subjects with a 30-month follow-up. The three regimens of treatment are:

a) Dabigatran $110 \mathrm{mg}$ twice-daily treatment strategy: Dabigatran 110 mg bid plus P2Y12 inhibitor (clopidogrel 75-mg or ticagrelor 90 $\mathrm{mg})$.

b) Dabigatran $150 \mathrm{mg}$ twice-daily treatment strategy: Dabigatran $150 \mathrm{mg}$ bid plus P2Y12 inhibitor (clopidogrel 75-mg or ticagrelor 90 $\mathrm{mg})$.

c) Dose-adjusted VKA treatment strategy: VKA (INR 2.0-3.0) and dual antiplatelet therapy with P2Y12 inhibitor (clopidogrel $75 \mathrm{mg}$ or ticagrelor $9 \mathrm{mg}$ ) plus low-dose aspirin.

In this study, there exist two primary endpoints. The primary safety endpoint will be determined by comparing a composite of the rate of overall death, MI, stroke or systemic embolism. The primary safety endpoint will compare the rate of major bleeding using the ISTH classification. Secondary endpoints include the rate of the composite endpoint of all-cause death, MI or stroke, as well as the following individual endpoints: all-cause death, cardiovascular death, noncardiovascular death, MI, stroke, systemic embolism, stent thrombosis and repeat revascularization (Table 2). 


\begin{tabular}{|c|c|c|c|}
\hline & PIONEER AF-PCI & RT-AF & REDUAL-PCI \\
\hline NOAC & Rivaroxaban & Rivaroxaban & Dabigatran Etexilate \\
\hline Population & $\begin{array}{c}\mathrm{AF} \text { and } \mathrm{ACS} \text { or Stable Angina undergoing } \mathrm{PCl} \\
+ \text { Stent (BMS or DES) }\end{array}$ & $\begin{array}{c}\text { AF and ACS or Stable Angina undergoing } \\
\text { PCI + Stent (BMS or DES) }\end{array}$ & $\begin{array}{c}\mathrm{AF} \text { and } \mathrm{ACS} \text { or Stable Angina undergoing } \mathrm{PCl}+ \\
\text { Stent (BMS or DES) }\end{array}$ \\
\hline Treatment arms & $\begin{array}{l}\text { a) Rivaroxaban 15-mg qd + Clopidogrel or } \\
\quad \text { Ticagrelor or Prasugrel } \\
\text { b) Rivaroxaban 2.5-mg bid + Clopidogrel or } \\
\text { Ticagrelor or Prasugrel + ASA } \rightarrow \text { Rivaroxaban } \\
\quad 2.5-\mathrm{mg} \text { bid + ASA } \\
\text { c) VKA (INR 2-3) + Clopidogrel or Ticagrelor } \\
\text { or Prasugrel + ASA } \rightarrow \text { VKA (INR 2-3) + ASA }\end{array}$ & $\begin{array}{l}\text { a) Rivaroxaban } 2.5 \text { or 5-mg bid + Ticagrelor } \\
\text { b) VKA (INR 1.8-2.5) + Clopidogrel + ASA }\end{array}$ & $\begin{array}{l}\text { a) Dabigatran 110-mg bid + Clopidogrel or } \\
\text { Ticagrelor } \\
\text { b) Dabigatran 150-mg bid + Clopidogrel or } \\
\text { Ticagrelor } \\
\text { c) VKA (INR 2-3) + Clopidogrel or Ticagrelor + ASA }\end{array}$ \\
\hline Follow-up & 12 months & 12 months & 30 months \\
\hline Sample size $(n)$ & 2100 & 420 & 8520 \\
\hline Primary endpoints & $\begin{array}{c}\text { - Safety: Composite of TIMI major bleeding, } \\
\text { bleeding requiring medical attention, and } \\
\text { minor bleeding }\end{array}$ & $\begin{array}{l}\text { - Safety: Composite of ISTH major bleeding } \\
\text { or clinically relevant non-major bleeding }\end{array}$ & $\begin{array}{c}\text { - Efficacy: Composite of all-cause death, MI, stroke } \\
\text { or systemic embolism } \\
\text { - Safety: ISTH major bleeding }\end{array}$ \\
\hline Study characteristics & $\begin{array}{l}\text { Randomized } \\
\text { Intention-to-treat } \\
\text { Open label }\end{array}$ & $\begin{array}{l}\text { Randomized } \\
\text { Intention-to-treat } \\
\text { Open label }\end{array}$ & $\begin{array}{l}\text { Randomized } \\
\text { Intention-to-treat } \\
\text { Open label }\end{array}$ \\
\hline
\end{tabular}

Table 2: Comparison in design of the PIONEER AF-PCI, the RT-AF and the RE-DUAL PCI trials. NOAC: Novel Oral Anticoagulants; AF: Atrial Fibrillation; ACS: Acute Coronary Syndromes; PCI: Percutaneous Coronary Intervention; BMS: Bare Metal Stent; DES: Drug-Eluting Stent; qd: once daily; bid: twice daily; VKA: Vitamin-K Antagonists; ASA: Aspirin; INR: International Normalized Ratio; TIMI: Thrombolysis in Myocardial Infarction; ISTH: International Society of Thrombosis and Hemostasis; MI: Myocardial Infarction.

\section{Conclusion}

ACS and AF have become cardiovascular epidemics in the twentyfirst century. Because both conditions present the thrombosis as central axis, long-term therapy with NOAC can operate as a cardinal therapy in patients with concomitant ACS and AF.

The feasibility of long-term therapy with NOAC was demonstrated as a result of studies with Ximelagatran. The safety of fixed dosing without routine coagulation monitoring is the key point for the treatment with IDT or FXa inhibitors. The hepatotoxicity of Ximelagatran has not been observed with other NOAC, enabling these new drugs have been evaluated in AF and ACS settings.

The PIONEER AF-PCI (Rivaroxaban), the RT-AF (Rivaroxaban) and the REDUAL-PCI (Dabigatran) trials may shed light on the safety and efficacy of long-term therapy with these new drugs in patients simultaneously presenting both diseases.

\section{References}

1. Bassand JP, Hamm CW, Ardissino D, Boersma E, Budaj A, et al. (2007) The task force for the diagnosis and treatment of non-ST-segment elevation acute coronary syndromes of the European Society of Cardiology. Guidelines for the diagnosis and treatment of non-ST-elevation acute coronary syndromes. Eur Heart J 28: 1598-1660.

2. Wallentin L, Becker RC, Budaj A, Cannon CP, Emanuelsson $\mathrm{H}$, et al. (2009) Ticagrelor versus clopidogrel in patients with acute coronary syndromes. $\mathrm{N}$ Engl J Med 361: 1045-1057.

3. Smith $P$, Arnesen H, Holme I (1990) The effect of warfarin on mortality and reinfarction after myocardial infarction. N Engl J Med 323: 147-152.

4. Hurlen M, Abdelnoor M, Smith P, Erikssen J, Arnesen H (2002) Warfarin, aspirin, or both after myocardial infarction. N Engl J Med 347: 969-974.

5. Dewilde WJ, Oirbans T, Verheugt FW, Kelder JC, De Smet BJ, et al. (2013) Use of clopidogrel with or without aspirin in patients taking oral anticoagulant therapy and undergoing percutaneous coronary intervention: An open-label, randomised, controlled trial. Lancet 381:1107-1115.

6. Bauer KA (2011) Recent progress in anticoagulant therapy: Oral direct inhibitors of thrombin and factor Xa. J Thromb Haemost 9 Suppl 1: 12-19.

7. Andreotti F, Pafundi T (2010) New anticoagulants and the future of cardiology. Rev Esp Cardiol 63: 1223-1229.

8. Wallentin L, Wilcox RG, Weaver WD, Emanuelsson H, Goodvin A, et al. (2003) Oral ximelagatran for secondary prophylaxis after myocardial infarction: the ESTEEM randomised controlled trial. Lancet 362: 789-797.
9. Mohapatra R, Tran M, Gore JM, Spencer FA (2005) A review of the oral direct thrombin inhibitor ximelagatran: Not yet the end of the warfarin era. Am Heart J 150: 19-26.

10. Oldgren J, Budaj A, Granger CB, Khder Y, Roberts J, et al. (2011) Dabigatran vs. placebo in patients with acute coronary syndromes on dual antiplatelet therapy: A randomized, double-blind, phase II trial. Eur Heart J 32: 2781-2789.

11. Dans AL, Connolly SJ, Wallentin L, Yang S, Nakamya J, et al. (2013) Concomitant use of antiplatelet therapy with dabigatran or warfarin in the Randomized Evaluation of Long-Term Anticoagulation Therapy (RE-LY) trial. Circulation 127: 634-640

12. Alexander JH, Lopes RD, James S, Kilaru R, He Y, et al. (2011) Apixaban with antiplatelet therapy after acute coronary syndrome. N Engl J Med 365: 699-708.

13. Steg PG, Mehta SR, Jukema JW, Lip GY, Gibson CM, et al. (2011) RUBY-1: A randomized, double-blind, placebo-controlled trial of the safety and tolerability of the novel oral factor Xa inhibitor darexaban (YM150) following acute coronary syndrome. Eur Heart J 32: 2541-2554.

14. Mega JL, Braunwald E, Wiviott SD, Bassand JP, Bhatt DL, et al. (2012) Rivaroxaban in patients with a recent acute coronary syndrome. N Engl J Med 366: 9-19.

15. Gibson CM, Mehran R, Bode C, Halperin J, Verheugt F, et al. (2015) PIONEER AF-PCl. Rivaroxaban and a dose-adjusted oral vitamin $\mathrm{k}$ antagonist treatment strategy in subjects with atrial fibrillation who undergo percutaneous coronary intervention. Am Heart J 169: 472-478.e5.

16. Gao F, Shen H, Wang ZJ, Yang SW, Liu XL, et al. (2015) Rationale and design of the RT-AF study: Combination of rivaroxaban and ticagrelor in patients with atrial fibrillation and coronary artery disease undergoing percutaneous coronary intervention. Contemp Clin Trials 43: 129-132.

17. Chesebro JH, Knatterud G, Roberts R, Borer J, Cohen LS, et al. (1987) Thrombolysis in Myocardial Infarction (TIMI) Trial, Phase I: A comparison between intravenous tissue plasminogen activator and intravenous streptokinase. Clinical findings through hospital discharge. Circulation 76: 142154.

18. Schulman S, Kearon C (2005) Subcommittee on Control of Anticoagulation of the Scientific and Standardization Committee of the International Society on Thrombosis and Haemostasis. Definition of major bleeding in clinical investigations of antihemostatic medicinal products in non-surgical patients. $J$ Thromb Haemost 3: 692-694

19. Boehringer Ingelheim (2014) Evaluation of Dual Therapy with Dabigatran vs Triple Therapy With Warfarin in Patients With AF That Undergo a PCI With Stenting (REDUAL-PCI). In: ClinicalTrials.gov. Bethesda (MD): National Library of Medicine, USA 